\title{
Viewing Your Kapwa: Elaboration of a Social-Relational Construct through Language
}

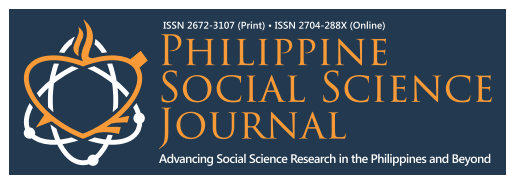

Paul Danielle P. Labor ${ }^{1}$ and Maria Cecilia C. Gastardo-Conaco ${ }^{2}$

1,2University of the Philippines, Diliman, Quezon City, Philippines

\section{Article history:}

Submitted: 4 September 2021

Revised: 23 November 2021

Accepted: 21 December 2021

\section{Keywords: \\ Kapwa \\ Language \\ Relational construal \\ Psychometrics \\ Sikolohiyang Pilipino}

ABSTRACT. Enriquez (1978) presented Kapwa as a core social psychological construct that could underpin Filipino social perception and interaction. However, issues arose regarding its conceptual and operational clarity as well as lack of empirical support. This two-phased study aims to address the issues presented through a psychometric approach. The first phase aimed to uncover construct characteristics by asking participants to provide definitions and examples of kapwa and di-kapwa, which were then examined via content analysis and the Linguistic Inquiry and Word Count (LIWC) program. Results from this phase elicited four (4) themes indicating that people viewed their social other in terms of dispositional attribution, level of interaction, connection, and inclusion. The second phase utilized this data to develop a kapwa measure which was found to have three (3) dimensions: Relations with the Social Other, Perceived Negative Characteristics, and Difference from the Self. Initial reliability and validity tests were favorable. Implications on Filipino social perception and behaviors highlight that we view our social others in terms of the aforementioned three dimensions and how differentially we might treat them depending on whether they are perceived as kapwa or di-kapwa. Furthermore, the results of the study further elaborate and nuance kapwa, which adds to theorizing in Sikolohiyang Pilipino (SP).

\subsection{Introduction}

Language is a resource in person perception. Classic studies indicate that an observer can infer another's social category (Champoux-Larsson, 2013; Ellis, 1967), age (Kent \& Burkard, 1981), or emotional state (Barrett et al., 2007; Lindquist \& Gendron, 2013) based on dialect, accent, or phonation. Recent studies show the impact of language and its use in the gendered-ness of political tweets (Hu \& Kearney, 2021), the influence of COVID-19 communications on adherence to health policies (Tu et al., 2021), biased punitive punishments for foreign-accented defendants (RomeroRivas et al., 2021), and language use as a possible indicator of suicide risk (Sierra et al., 2021). These highlight language as a social cue that allows people to calibrate their behavior, allowing for the regulation of relationship processes through modes of address and reference (Brown \& Gilman, 1960) and verbal and non-verbal actions utilized (Robinson, 2008).

In the Philippines, a construct implicated in person perception and interpersonal relations is Kapwa, usually translated as 'other' (English, 1977). In the area of Sikolohiyang Pilipino (SP: Filipino Psychology), it was theorized as a sense of 'shared identity', or 'unity of the "self" and "others"' (Enriquez, 1978, p. 28). It was recognized as a core value in Enriquez's (1992) theorizing of Filipino behavioral and value structure. As a superordinate construct, it encompasses individuals belonging to one's in-groups and out-groups. However, closeness to an individual lies within an eight (8) level social interaction continuum with civility for an outsider on one end, leading to unity with a significant-close other (Santiago \& Enriquez, 1976).

However, Enriquez's theory has been under scrutiny due to the amorphous nature of kapwa. In his seminal publication, it was characterized as a social interaction construct that meant the "unity of the self and others" or a "recognition of shared identity" (Enriquez, 1978, p. 28). Yet, in the same paper, kapwa is also characterized as a "core value" (Enriquez, 1978, p. 27). A subsequent publication also defines kapwa in both ways (Enriquez, 1992). Criticisms have pointed out the lack of contextualization for kapwa (Sta. Maria, 1996) and the seeming lack of basis for the constructs that comprise the overall theory (Clemente et al., 2008). This ambiguity has led other researchers to diverge from Enriquez's original formulation and conduct studies where kapwa is defined either

This article published by Philippine Social Science Journal (PSSJ) is licensed under a Creative Commons AttributionNoncommercial 4.0 International (CC BY-NC 4.0). You are free to share (copy and redistribute the material in any medium or format) and adapt (remix, transform, and build upon the material). Under the following terms, you must give appropriate credit, provide a link to the license, and indicate if changes were made. You may do so in any reasonable manner, but not in any way that suggests the licensor endorses you or your use. You may not use the material for commercial purposes. 
as a social-relational construct (Gastardo-Conaco, 2009; Gastardo-Conaco \& Ortega, 2011) or value (Clemente et al., 2008; Yacat, 2017).

These criticisms provide further impetus for studies on the theoretical elaboration of kapwa. This study addresses the gap in theoretical clarity and lack of a viable measure by undertaking a language and psychometric approach. It aims to describe the characteristics of the construct via the words utilized by the participants who try to define it. A linguistic analysis, specifically the Linguistic Inquiry and Word Count (Pennebaker et al., 2015; Pennebaker et al., 2007; Tausczik \& Pennebaker, 2010), will be utilized in line with this goal. Then, a measure will be developed through exploratory factor analysis to identify possible underlying dimensions. This scale's relationship with other similar constructs will also be determined to establish reliability and validity. This can further shed insight into the nature of kapwa as an important variable in Filipino social relationships.

\subsection{Framework of the Study}

The study seeks to address the gap presented by the kapwa construct's conceptual vagueness and lack of a viable measure through a psychometric approach informed by language. Psychometrics is concerned with developing scales and their evaluation in terms of the information they provide, their reliability, and their validity (Furr \& Bacharach, 2014). Through scale development, a construct's operationalization is clarified, underlying dimensions identified, with the resulting scale used to effectively distinguish between individuals who highly possess or endorse the construct from those who do not. This approach is utilized to further explore kapwa. Past work has contributed to expounding on more empirically grounded kapwa conceptualizations and processes. These works utilized either Enriquez's original value structure theory (Enriquez, 1992) or approached it as a socialrelational construct (Gastardo-Conaco, 2009; Gastardo-Conaco \& Ortega, 2011). Their insights provide the groundwork and inform the psychometric approach taken in this study.

Studies utilizing Enriquez's original theorizing defined kapwa as a value but failed to provide a reliable and valid measure. Clemente et al. (2008) revisited Enriquez's theory (1992) to determine whether the values previously presented were still currently endorsed and whether a core value would emerge. Results indicated that only 11 of the original 12 values were endorsed, and kapwa did not surface as a core value. Yacat (2017) explored the role of value violator categorization (i.e., acquaintance/ibangtao versus friend/hindi-ibang-tao) on violation severity perceptions, negative affect, and relationship maintenance. Pertinent to Kapwa theory, his results confirm the initial tenets that even though all individuals are deemed kapwa, how we interact is bound to whether one is categorized as ibang-tao or hindi-ibang-tao (Enriquez, 1978; Santiago, 1976).

Social-relational kapwa studies contributed to the initial psychometric approach the present study builds on. Gastardo-Conaco (2009) showed how the categorization of the social other as kapwa or dikapwa influenced the goals of pakikiramdam, which underlines the role of categorization in determining how we interact with others. But critically, she provides pakikiramdam as the mechanism by which this occurs. Gastardo-Conaco and Ortega (2011) elaborate on kapwa processes and characteristics by finding five (5) components within a valid and reliable scale: similarity cognitions, relational links, positive behaviors, negative behaviors, and positive affect. Furthermore, they show that although demarcations between in-groups and out-groups are recognized, the sense of kapwa extends beyond one's in-group and operates even towards outsiders.

The studies detailed inform that kapwa seems to encompass both in-groups and out-groups. However, that is qualified by the type of interaction one implements depending on how close the other is. Another mechanism by which it operates is pakikiramdam or shared inner perception, wherein interaction goals depend on whether the other is kapwa or not. Although ultimately, the resulting interaction is meant to be positive and non-detrimental to the parties involved.

But what is kapwa truly? The divergent theoretical threads indicated do not resolve questions on kapwa's nature. A stringent psychometric approach should resolve this. Eliciting kapwa conceptualizations will elucidate whether it is a value, social-relational construct, or something else. Items developed from these responses will undergo greater inspection in terms of their nature and dimensionality. Although this was initially done by Gastardo-Conaco and Ortega (2011), their strategy of developing scale components through direct inspection of item similarities did not allow for identifying underlying kapwa dimensions that could otherwise be identified through exploratory factor analysis (Floyd \& Widaman, 1995). Finally, ascertaining kapwa's nature through comparison with similar socialpsychological constructs will further clarify its operationalization with a reliable and valid measure. 


\subsection{Methods}

The study's research design comprises two phases. Phase 1 will provide the conceptual handles on kapwa. Similar to Piamonte et al. (2020) and Lopena et al. (2021), we utilized a qualitative approach to surface interpersonal connections and experiences within a social context. Participants are asked to define both kapwa and di-kapwa and provide examples for both. Responses also provide an inkling as to whether kapwa is a value or social-relational construct. Results from Phase 1 provide the backbone for the Phase 2 scale development. Based on the responses and themes elicited from Phase 1, items for the kapwa scale are constructed. These items then undergo exploratory factor analysis to identify underlying dimensions. With dimensionality clarified, the scale undergoes reliability and validity tests. This, ultimately, will answer the questions regarding kapwa's nature and possible processes.

\section{Phase 1}

Participants. A total of 617 participants of varying backgrounds and age groups participated. In this non-probability convenience sample, participants were from 14 to 77 years old; two-thirds were female, with a mean age of 29.77 years $(s d=13.86)$.

Procedure.Participants responded to a Google Forms survey asking them to elaborate on kapwa and di-kapwa. They were prompted to continue the following sentences and give examples:

(1) Para sa akin, ang kapwa ay ...'

a. 'Magbigay ng halimbawa.'

(2) 'Para sa akin, ang di-kapwa ay ...'

a. 'Magbigay ng halimbawa.'

Yoshikoder(Lowe, 2015) was utilized to provide the initial word counts of the 2468 kapwa and dikapwa responses. For the raw word count of the original Filipino responses, there were 4641 words for kapwa definitions, 5824 words for kapwa examples, 3599 words for di-kapwa definitions, and 6770 words for di-kapwa examples. Thereafter, the responses were translated into English. First, the responses were run through Google Translate for an initial rough translation. Three (3) native Filipinospeaking psychology graduate students were hired as translators. They independently checked the English translations for accuracy to ensure essence and equivalence. They checked each other's work and discussed any conflicting translations to reach a consensus. Participants' verbal responses were then placed in 20 "bags of words" for kapwa conceptualizations, kapwa examples, di-kapwa examples, and di-kapwa conceptualizations divided into overall bags for each category, gender (i.e., male and female) and age group (i.e., $\leq 24$ years old and $\geq 25$ years old).

The translated texts were then analyzed through LIWC (Pennebaker et al., 2015; 2007; Tausczik \& Pennebaker, 2010), which taps into meaningful psychological features present in linguistic corpora to provide information regarding an individual's cognitive and affective landscape. About 80 categories detail various psychological features such as affective, cognitive, and biological processes, drives, time orientations, and personal concerns. To date, LIWC has shown great reliability across time (Pennebaker et al., 2015; 2007). These procedures were performed to collect greater descriptive information on the kapwa construct.

\section{Phase 2}

Participants.Participants were 306 college students coming from a university located in Metro Manila. Two-thirds of the sample were female with a mean age of 19.01 years (sd $=1.85$ ).

Measures.Kapwa scale. Items generated were based on the themes and participants' responses from the first phase. The four assumed dimensions had 3-12 items each: dispositional attribution (12-items), connection (5-items), interaction (3-items), and inclusion (5-items) for a total of 25 items.

Self-Construal. Singelis' (1994) 30-item Self-Construal Scale measures an individual's view of the self - whether independent or interdependent of others. It has two subscales composed of 15 -items each. Independence and interdependence subscales had acceptable (Cronbach's $\alpha$ ) reliabilities of .71 and .63 , respectively.

Inclusion of the Other. The Inclusion of the Other in the Self Scale (IOS) by Aron et al. (1992) is a single-item measure of closeness. Four IOS iterations were made with different referents: Mother, Neighbor, Filipino, and American. 
Procedure and Design.Participants responded to a questionnaire containing items from the Kapwa, Self-Construal, and IOS scales, as well as demographic questions on age, gender, year-level, and degree. The Kapwa scale was subjected to exploratory factor analysis utilizing principal axis factoring with direct oblimin rotation. The rotation allows for correlation among factors especially appropriate for psychological constructs (Pett et al., 2003) as orthogonal rotations may not portray psychological constructs realistically (Pedhazur \& Schmelkin, 2013). Lastly, the kapwa scale underwent reliability analysis and initial validity tests by determining its relationship with self-construal and IOS.

Ethical Considerations. In both phases of the study, ethical standards were strictly followed. At the onset, participants on both phases were informed that their participation was fully voluntary and that the study was exceedingly low-risk as its topic was on social perceptions. If they chose to participate and at any point decided to stop, they would be able to do so without any ramifications. The demographic information provided would be kept confidential to ensure anonymity. Furthermore, responses would be analyzed at the group level where no one would be a singular focus. Data collected from both phases would only be handled by the two researchers, kept in secure hard drives, and destroyed after an appropriate amount of time. Contact details of the researchers were provided if participants wanted to provide feedback, raise concerns, or withdraw their data at any point. All of these were detailed in informed consent forms the participants read before signing to participate.

\subsection{Results}

\section{Phase 1}

We first looked at the examples. On kapwa, words with the highest proportions were all nouns and pronouns (99\%) pertaining to social categories and people (e.g., kaibigan, pamilya, kapitbahay). Noticeable in these is the outright use of prefixes (17\%) indicative of relationship or similarity such as taga-, kapwa-, taong- or tao-sa, and ka-. Conversely, di-kapwa majority words (18\%) were negative attributes (i.e., masama, makasarili, mayabang) and behaviors (i.e., sinasaktan, binabastos, inaapi). Several also had negatory prefixes (6\%) such as hindior di-, and wala or walang.

Di-kapwa responses were observed as opposites of the kapwa ones. This mirroring effect was seen in participants' elaboration of those considered as di-kapwa. This supposes that the elicited themes pertinent to kapwa may also be applied to the di-kapwa - but endorsed in the opposite manner. That is the dispositional attributions are mostly negative (e.g., masama, makasarili, dimapagkakatiwalaan). The level of interaction and connection is low and/or highly negative (i.e., kaaway, paghihiwalay-hiwalay, ayaw makisalamuha), and level of inclusion is weak (i.e. ibang tao, limitado sa tao, parepareho).

LIWC analyses focused on dimensions of emotional tone, pronouns, and personal pronouns, social processes, affiliation, and focus (i.e., present). Results highlight the social aspect of kapwa. Across gender and age groups (i.e. $\leq 24$ years $\& \geq 25$ years), the general emotional tone (score $\leq 49$ $=$ negative, score $\geq 50$ = positive) for kapwa conceptualizations (score $=93.00$ ) and examples (score $=49.71$ ) were more or less positive - while being negative in di-kapwa data (conceptualizations score $=1.00$; examples score $=26.74$ ). Moreover, there was significant usage of pronouns and personal pronouns (i.e., roughly $17.53 \%$ of total words across kapwa and di-kapwa data), indicating a salience and focus to self and others (Pennebaker et al., 2003; Tausczik \& Pennebaker, 2010). This is further supported by the percentage of words that fall into the social processes dimension (i.e., 20-30\%) in kapwa and di-kapwa conceptualizations and examples across gender and age groups.

In the drive for affiliation dimension, lower percentages (i.e., 2-5\%) were found for di-kapwa conceptualizations and examples compared to kapwa (i.e., 9-14\%) ones across gender and age groups. This indicates that participants' affiliative needs were not fulfilled by the di-kapwa, while belongingness and involvement needs were seen more in kapwa-type relations. Finally, the data indicate that both kapwa and di-kapwa conceptualizations and examples generally utilize a significant percentage of present-tense verbs (i.e., 11-14\%), indicating that both processes are situated in immediate events.

Content analysis of participants' responses to the prompt questions on kapwa was partly guided by Jarymowicz's (2015) different types of 'We' concepts. 'We' concepts are based on group identity (i.e., based on real experience and direct contact), categorical identity (i.e., social or ethnic identities), attributive (i.e., personal traits), and axiological identity (i.e., human beings). We utilized these as initial guideposts in determining how participants viewed those whom they identified as 
kapwa. Furthermore, we utilized Braun and Clarke's $(2006,2013)$ procedures for eliciting themes from qualitative data: familiarizing yourself with the data, generating initial codes, searching for themes, reviewing themes, defining and naming themes, and producing the report. After we familiarized ourselves with the data, results from the initial word count and LIWC acted as initial codes. The frequency, type, and subjects the words pertained to showed interesting and common features. These allowed for the identification of initial themes which were reviewed. The themes were finalized, named, and defined through a discussion to reach a concurrence from both authors. The responses to the questions, the examples provided, word-count, and LIWC results were the bases for the themes:

- Dispositional attribution: Kapwa was ascribed with positive personal traits such as being matulungin, maasahan,mabait, mapagkalinga, maymalasakit, among others.

- Level of interaction: Kapwa was someone one had regular interactions with (e.g., laging nakakasalamuha, mga nakakasalamuha, taong nakapaligid). Examples mentioned social categories like family, friends, and acquaintances where regular or occasional social interaction is characteristic.

- Connection: This focuses on the link or relations one has with the kapwa. Relationship examples given by the participants were those that had moderate to strong social bonds such as family and friends.

- Inclusion: This focused on the perceived degree of social inclusion of those deemed as kapwa. Responses highlighted qualifiers used by participants to demarcate inclusion and exclusion, such as lahat or kahit sino. Other qualifiers underscored similarity (kapareho) and degree of knowing (kakilala o di-kakilala).

\section{Phase 2}

\section{Factor Structure}

Initial tests for acceptability of the data for exploratory factor analysis (EFA) yielded favorable results. Kaiser-Meyer-Olkin (KMO $=.94, \mathrm{n}=300$ ) statistics indicated decent sampling adequacy relative to the number of items in the scale. Bartlett's Test of Sphericity confirmed that the correlation matrix was not an identity matrix, $\chi 2(300)=5280.26, p<.0001$, which indicated the non-violation of EFA assumptions.

Findings from the scree-plot and Kaiser-Guttman rule (Nunnally \& Bernstein, 1994) indicate a three (3) factor solution accounting for $56.47 \%$ of the variance. The three kapwa dimensions found were Relations with the Social Other (RSO), Perceived Negative Characteristics (PNC), and Difference from the Self (DFS) (see Table 1). The three factors were significantly correlated $(p<.001)$ with each other with RSO and PNC correlating at $r=-.50$, RSO and DFS at $r=.70$, and PNC and DFS at -.31 . Based on the factor structure, kapwa may be defined and operationalized as a social other an individual perceives to have high levels of interaction and connection. A kapwa is someone viewed to have positive characteristics that they perceive to be greatly similar to themselves.

\section{Reliability and Validity}

The subscales on Relations with the Social Other (RSO), Perceived Negative Characteristics (PNC), and Difference from the Self (DFS) had reliability coefficients (Cronbach's alpha) of .95, .74, and .85 , respectively. Item no. 12 of the second factor was removed to further increase subscale reliability as it differentially cross-loaded in both RSO and PNC - resulting in a coefficient of 0.85 for the 9-item PNC subscale. The 22-item Kapwa Scale had an overall reliability coefficient of 0.81 . These indicate favorable reliability for the Kapwa Scale.

Initial tests of the scale's validity were performed through a correlation analysis. Theoretically, the RSO and DFS subscales should positively correlate with interdependent self-construal and the four Inclusion of the Other in the Self Scale (IOS) referents as all these measures are about viewing others vis-a-vis the self. Conversely, there should be weak to no correlations between independent selfconstrual and the RSO and DFS subscales as the former measure focuses more on a very demarcated view of the self, unlike kapwa. No significant correlations are expected between the PNC subscale and the self-construal or closeness measures as the former is other-focused and not necessarily related to closeness and views on the self. 
Table 1. Summary Structure Matrix and Factor Loadings

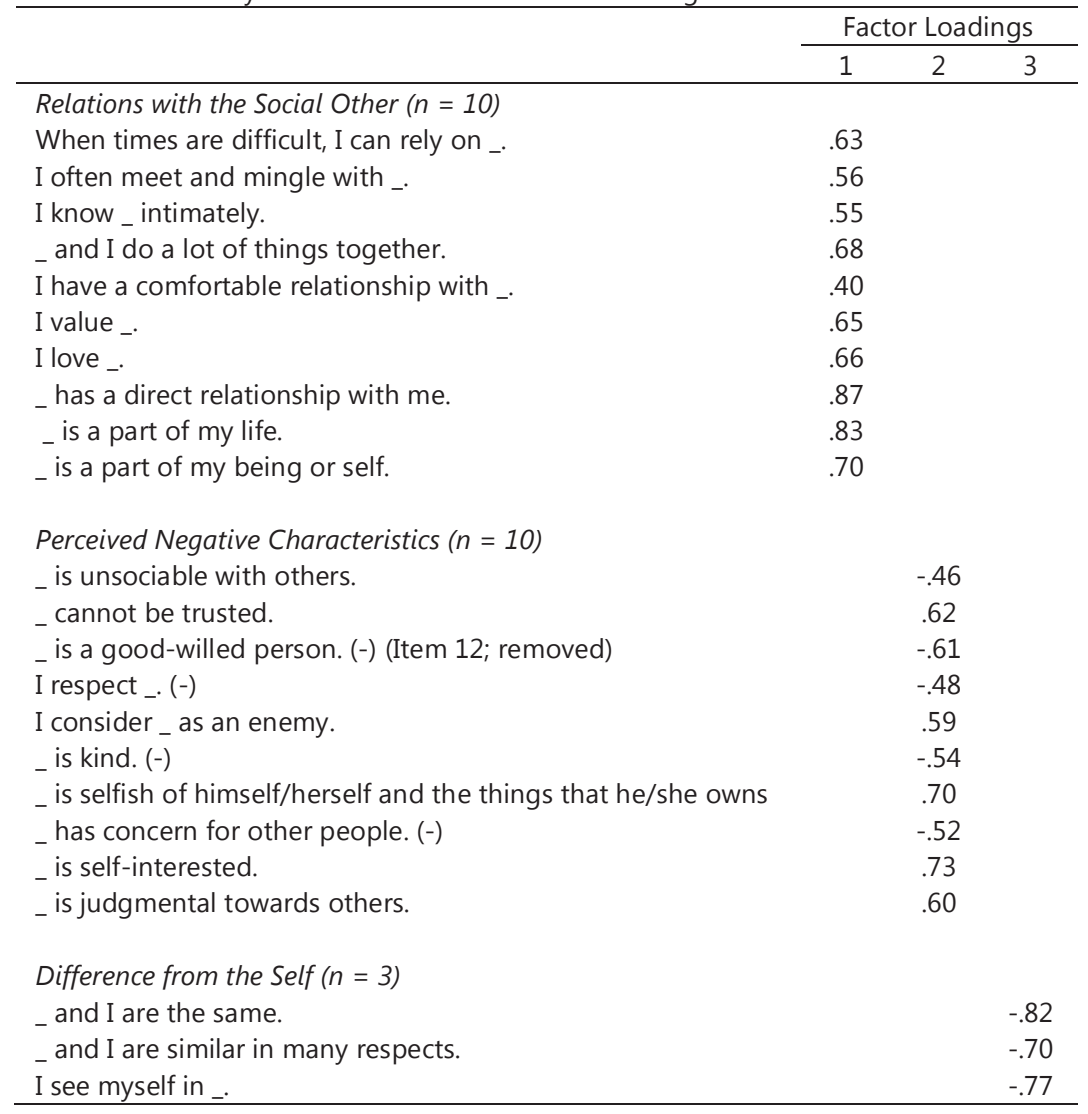

Table 2. Correlations

\begin{tabular}{|c|c|c|c|c|c|c|c|c|c|}
\hline & 1 & 2 & 3 & 4 & 5 & 6 & 7 & 8 & 9 \\
\hline 1. RSO subscale & - & $-.50^{\star *}$ & $.70^{* *}$ & .08 & $.22^{* *}$ & $.15^{* *}$ & $.17^{\star \star}$ & $.21^{\star *}$ & $.17^{* *}$ \\
\hline 2.PNC subscale & & - & $-.31^{\star *}$ & -.05 & $-.25^{\star \star}$ & $-.16^{\star \star}$ & -.10 & $-.15^{\star \star}$ & -.04 \\
\hline 3. DFS subscale & & & - & .10 & $.18^{\star \star}$ & $.17^{\star \star}$ & $.18^{* *}$ & $.19 * \star$ & $.19 * *$ \\
\hline 4. Independent & & & & - & .07 & $.12^{\star}$ & $.12^{*}$ & $.14^{*}$ & $.17^{\star *}$ \\
\hline 5. Interdependent & & & & & - & $.23^{* *}$ & $.15^{* *}$ & $.19 * *$ & .07 \\
\hline 6. IOS: Mother & & & & & & - & $.37^{\star *}$ & $.43^{\star \star}$ & $.40 * *$ \\
\hline 7. IOS: Neighbor & & & & & & & - & $.43^{* *}$ & $.36^{* *}$ \\
\hline 8. IOS: Filipino & & & & & & & & - & $.51^{* *}$ \\
\hline 9. IOS American & & & & & & & & & - \\
\hline
\end{tabular}

The results of the correlational analysis provide initial validity to the Kapwa subscales. Both RSO and DFS subscales were positively correlated with interdependent self-construal and the four IOS referents. Contrariwise, the PNC subscale was negatively correlated with interdependent self-construal and the four IOS items. All the Kapwa subscales did not correlate significantly with independent self- 
construal. Finally, correlations between the kapwa subscales and validation measures were weak to moderate. This indicates shared variance among the measures but not enough to say that the kapwa subscales were no different from the validation measures. There is enough unique variance in the kapwa subscales to conclude that the scale differs from other similar measures. These provide initial convergent and discriminant validity for the Kapwa scale.

\subsection{Discussion}

We aimed to address conceptualization and operationalization issues of the kapwa construct proposed by Enriquez $(1978,1992)$. Findings from the first phase indicate kapwa as about social categories such as family, friends, and other people one knows. Conversely, di-kapwa were individuals viewed to have negative characteristics and performed objectionable behaviors. From these examples and definitions, four (4) themes were extracted: dispositional attribution, connection, level of interaction, and inclusion. These highlight kapwa as a social construal of the other. Subsequently, a three-factor measure was developed: relations with the social other (RSO), perceived negative characteristics (PNC), and difference from the self (DFS). These coincide with the themes found during the first phase; except, for the RSO dimension, which seems to be a combination of the connection and level of interaction themes. This kapwa scale was then tested for reliability and validity and was found to be satisfactory.

The findings from this study were consistent with prior work done by Gastardo-Conaco (2009) and Gastardo-Conaco and Ortega (2011), which operationalized kapwa as a social-relational construct in contrast to the original theorizing of Enriquez $(1978,1992)$. However, the DFS dimension found in the current study supports Enriquez's earlier definitions of kapwa as "unity of the self and others" or a "recognition of shared identity" (Enriquez, 1978, p. 28). The current study, utilizing a bottom-up approach through language similar to grounded theory (Glaser \& Strauss, 2017), surfaced a more nuanced definition of kapwa. Not only is kapwa a "unity of the self and others" or "recognition of shared identity" (Enriquez, 1978, p. 28) through the DFS dimension. It is a view of a highly positive social other to which one has strong connections and interactions, lending robust empirical support to the notion of kapwa as a lens through which we view our social others.

But where does this place the theory of kapwa as value? Work by Clemente et al. (2008) and Yacat (2017), clarified and differentiated kapwa from pakikipagkapwa. In both papers, it is pakikipagkapwa that is deemed a value - not kapwa. The value of pakikipagkapwa is dedicated towards the recognition of the dignity and humane treatment of the social other (Yacat, 2017). These findings, in conjunction with the present study's, present a significant difference between the constructs. It denotes the presence of two significant constructs within Enriquez's Kapwa theory: a social perception construct in kapwa and a value in pakikipagkapwa. However, what is unclear is how these constructs work together. The dynamics between kapwa and pakikipagkapwa have significant implications on Kapwa theory. Further empirical work may update Kapwa theory and add to our understanding of Filipino social behavior.

Another important finding from the study is the recognition of di-kapwa as a separate social category from those earlier proposed in Enriquez's model (1992). For him, everyone was considered as kapwa - even outsiders (ibang-tao). However, results from the first phase of this study indicate dikapwa as a different category altogether. The di-kapwa are social others with negative characteristics and bad behaviors. Enemies, as well as moral and norm violators, come to mind when faced with this description. Furthermore, as di-kapwa are outside the kapwa boundaries, the modes of social interactions towards kapwa will not apply to the di-kapwa. Characteristically neutral to positive kapwa-type social interactions may not be how individuals behave when dealing with a di-kapwa. It is entirely possible and empirically testable that when dealing with a di-kapwa, one can be unpleasant or harsh. There are parallels to this in the area of moral exclusion wherein those outside the bounds of one's moral community are excluded and considered "nonentities, expendable, or undeserving" (Opotow, 1990, p. 1). As a result, harmful and unfair behavior towards them may be considered reasonable and acceptable. This has severe implications on individuals deemed as di-kapwa as we usually rely on social others to cope with stressful life events (Valladolid, 2021).

Finally, this empirically supported elaboration of kapwa as a social-relational construct has significant implications for Sikolohiyang Pilipino (SP). As an indigenous psychology, SP is focused on "psychology based on the experience, ideas, and orientation of the Filipino" (Enriquez, 1994, p. 3). It has seen great strides in advancing critical dialogue on the utility and applicability of foreign 
frameworks to the Filipino psyche. It has also elucidated and clarified many indigenous concepts. However, SP work has been criticized as being theoretically narrow and lacking empirical support (Church \& Katigbak, 2002). Enriquez's $(1978,1992)$ Kapwa theory was treated almost like an unshakeable and sacred canon that only a few ventured to critically challenge its tenets and provide empirical supports (Gastardo-Conaco, 2005). With this study's findings and the recent resurgence of SP work, revisiting and updating the theory based on empirical work and addressing the prior criticisms on SP as a whole may be addressed. For SP to strengthen, it also needs to address the many ills that face indigenous psychologies, such as the low workability and tenability of proposed theories and frameworks (Jahoda, 2016). SP has much to do. With this initial work and those of others, an updated Kapwa theory can provide new avenues for research and applied possibilities.

\subsection{Conclusion}

This work identifies fruitful areas of inquiry. First, further psychometric tests would be prudent to establish the soundness of the measure. As most of the work on kapwa has been done among the Tagalog groups, it would be beneficial and more inclusive to test it across different ethnolinguistic groups which have their cultural systems to which kapwa may not apply (Lindquist \& Gendron, 2013). Work along this line of inquiry would establish the construct's boundary conditions and also contribute to more inclusive theorizing on Filipino social perception and behavior.

The second line of work that emerged from this study is on the conceptualization and elaboration of di-kapwa social others. Initial findings from the first phase of this study characterize di-kapwa social others as distinct from kapwa. This conceptual cleavage opens up queries not only on conceptual clarity and operationalization but also on questions regarding social behavior directed to the di-kapwa. As mentioned in the discussion, parallel work from moral exclusion (Opotow, 1990) can give us glimpses of how the di-kapwa may be treated. However, empirical work is needed to establish the concept's operationalization and process as this has significant implications on the areas of aggression, altruism, and inclusion-exclusion.

\subsection{Acknowledgments}

Funding for this project came from a University of the Philippines Enhanced Creative Work and Research Grant (ECWRG) awarded to the second author in 2015.

\section{REFERENCES}

Aron, A., Aron, E. N., \& Smollan, D. (1992). Inclusion of others in the self scale and the structure of interpersonal closeness. Journal of Personality and Social Psychology, 63(4), 596-612. https://doi.org/10.1037/00223514.63.4.596

Barrett, L. F., Lindquist, K. A., \& Gendron, M. (2007). Language as context for the perception of emotion. Trends in Cognitive Sciences, 11(8), 327-332. https://doi.org/10.1016/j.tics.2007.06.003

Braun, V., \& Clarke, V. (2006). Using thematic analysis in psychology. Qualitative Research in Psychology, 3(2), 77-101. https://doi.org/https://doi.org/10.1191/1478088706qp063oa

Braun, V., \& Clarke, V. (2013). Successful qualitative research: A practical guide for beginners. London, England: SAGE Publications, Ltd.

Brown, R., \& Gilman, A. (1960). The pronouns of power and solidarity. In T. A. Sebeok (Ed.), Style in langauge (pp. 252-275). Cambridge, MA: MIT Press.

Champoux-Larsson, M. F., Ramström, F., Costa, A., \& Baus, C. (2013). Social categorization based on language and facial recognition. Journal of Language and Social Psychology. https://doi.org/10.1177/0261927X211035159

Church, T., \& Katigbak, M. S. (2002). Indigenization of psychology in the Philippines. International Journal of Psychology, 37(3), 129-148. https://doi.org/10.1080/0020759014300031

Clemente, J. A., Belleza, D., Yu, A., Catibog, E. V. D., Solis, G., \& Laguerta, J. (2008). Revisiting the kapwa theory: Applying alternative methodologies and gaining new insights. Philippine Journal of Psychology, 41(2), 1-32.

Ellis, D. S. (1967). Speech and social status in America. Social Forces, 45(3), 431-437. https://doi.org/10.1093/ sf/45.3.431

English, L. J. (1977). English-tagalog dictionary. Manila: Congregation of the Most Holy Redeemer.

Enriquez, V. G. (1978). Kapwa: A core concept in Filipino social psychology. Philippine Social Sciences and Humanities Review, 42(1-4), 100-108.

Enriquez, V. G. (1992). From colonial to liberation psychology: The Philippine experience. Quezon City: University of the Philippines Press.

Enriquez, V. G. (1994). Pagbabagong-dangal: Indigenous psychology and cultural empowerment. Quezon City: Akademya ng Kultura at Sikolohiyang Pilipino. 
Floyd, F. J., \& Widaman, K. F. (1995). Factor analysis in the development and refinement of clinical assessment instruments. Psychological Assessment, 7(3), 286-299. https://doi.org/10.1037/1040-3590.7.3.286

Furr, R. M., \& Bacharach, V. R. (2014). Psychometrics: An introduction (2nd Ed.). Los Angeles: Sage Publications Ltd.

Gastardo-Conaco, M. C. (2005). The development of a Filipino indigenous psychology. Philippine Journal of Psychology, 38(2), 1-17.

Gastardo-Conaco, M. C. (2009). Pakikiramdam and kapwa: Reading emotion in a personalistic culture. In R. Ismail, M. E. J. Macapagal, N. M. Noor, J. Takai, \& T. Hur (Eds.), Global issues and challenges in a changing world: Psychological, cultural, and group relationships (Progress in Asian Psychology, Vol. 7) (pp. 309-321). Malaysia: Center for Research and Innovation.

Gastardo-Conaco, M. C., \& Ortega, S. C. (2011). Assessing "kapwa": Setting the parameters of a Filipino relational construct. In P. Singh, P. Bain, C. Leong, \& Y. Ohtsubo (Eds.), Individual, group, and cultural processes in changing societies (Progress in Asian Psychology, Vol. 8) (pp. 278-288). Delhi: University Press.

Glaser, B. G., \& Strauss, A. L. (2017). Discovery of grounded theory: Strategies for qualitative research. Routledge.

Hu, L., \& Kearney, M. W. (2021). Gendered tweets: Computational text analysis of gender differences in political discussion on Twitter. Journal of Language and Social Psychology, 40(4), 482-503. https://doi. org/10.1177/0261927X20969752

Jahoda, G. (2016). On the rise and decline of 'indigenous psychology. Culture \& Psychology, 22(2), 169-181. https://doi.org/10.1177/1354067X16634052

Jarymowicz, M. (2015). Mental barriers and links connecting people of different cultures: Experiential vs. conceptual bases of different types of the we-concepts. Frontiers in Psychology, 6(December), 1-4. https:// doi.org/10.3389/fpsyg.2015.01950

Kent, R. D., \& Burkard, R. (1981). Change in the acoustical correlates of speech production. In D. S. Beasley \& G. A. Davis (Eds.). Aging: Communication Processes and Disorders (pp. 47-62). New York, NY: Grune \& Stratton.

Lindquist, K. A., \& Gendron, M. (2013). What's in a word? Language constructs emotion perception. Emotion Review, 5(1), 66-71. https://doi.org/10.1177/1754073912451351

Lopena, G. L., Padilla, N. D., \& Madrigal, D. V. (2021). Walking through a maze: The struggles of accountancy students with online learning in the context of the COVID-19 pandemic. Philippine Social Science Journal, 4(3), 30-38. https://doi.org/10.52006/main.v4i3.375

Lowe, W. (2015). Yoshikoder: Cross-platform multilingual content analysis. Java software version 0.6.5. http://www. yoshikoder.org

Nunnally, J. C., \& Bernstein, I. H. (1994). Psychometric theory (McGraw-Hill Series in Psychology) (Vol. 3). New York, New York: McGraw-Hill.

Opotow, S. (1990). Moral exclusion and injustice: An introduction. Journal of Social Issues, 46(1), 1-20. https://doi. org/10.1111/j.1540-4560.1990.tb00268.x

Pedhazur, E. J., \& Schmelkin, L. P. (2013). Measurement, design, and analysis: An integrated approach. Hillsdale: Lawrence Erlbaum Associates.

Pennebaker, J. W., Boyd, R., Jordan, K., \& Blackburn, K. (2015). The development and psychometric properties of LIWC2015. Auston, TX: University of Texas at Austin. https://doi.org/10.15781/T29G6Z

Pennebaker, J. W., Chung, C. K., Ireland, M., Gonzales, A., \& Booth, R. J. (2007). The development and psychometric properties of LIWC2007 [software manual]. University of Texas at Austin and The University of Auckland. https://doi.org/10.1068/d010163

Pennebaker, J. W., Mehl, M. R., \& Niederhoffer, K. G. (2003). Psychological aspects of natural language use: Our words, our selves. Annual Review of Psychology, 54, 547-577. https://doi.org/10.1146/annurev. psych.54.101601.145041

Pett, M. A., Lackey, N. R., \& Sullivan, J. J. (2003). Making sense of factor analysis: The use of factor analysis for instrument development in health care research. Thousand Oaks, California: SAGE Publications, Inc.

Piamonte, S. B. H., Quintos, M. A. M., \& Iwayama, M. O. (2020). An exploratory study on the practice of pagsasanto by openly-gay santeros. Philippine Social Science Journal, 3(3), 44-53. https://doi.org/10.52006/main. v3i3.275

Robinson, W. P. (2008). Language in social worlds. Great Britain: Blackwell Publishers Ltd.

Romero-Rivas, C., Morgan, C., \& Collier, T. (2021). Accentism on trial: Categorization/stereotyping and implicit biases predict harsher sentences for foreign-accented defendants. Journal of Language and Social Psychology. https://doi.org/10.1177/0261927X211022785

Santiago, C. E. (1976). The language of food. In G. Cordero (Ed.). Culinary Culture of the Philippines (pp. 133-139). Philippines: Bancom Audiovision Corporation.

Santiago, C. E., \& Enriquez, V. G. (1976). Tungo sa makaPilipinong pananaliksik. Sikolohiyang Pilipino: Mga Ulat at Balita, 1(4), 3-10.

Sierra, G., Andrade-Palos, P., Bel-Enguix, G., Osornio-Arteaga, A., Cabrera-Mora, A., García-Nieto, L., \& SierraAparicio, T. (2021). Suicide risk factors: A language analysis approach in social media. Journal of Language and Social Psychology, 1-19. https://doi.org/10.1177/0261927X211036171

Singelis, T. M. (1994). The measurement of independent and interdependent self-construals. Personality and Social Psychology Bulletin, 20(5), 580-591. https://doi.org/10.1177/0146167294205014

Sta. Maria, M. (1996). Is the indigenization crisis in the Philippine Social sciences resolved in sikolohiyang Pilipino. Layag, 1(1), 101-120. 
Tausczik, Y. R., \& Pennebaker, J. W. (2010). The psychological meaning of words: LIWC and computerized text analysis methods. Journal of Language and Social Psychology, 29(1), 24-54. https://doi. org/10.1177/0261927X09351676

Tu, K. C., Chen, S. S., \& Mesler, R. M. (2021). "We" are in this pandemic, but "you" can get through this: The effects of pronouns on likelihood to stay-at-home during COVID-19. Journal of Language and Social Psychology, 1-15. https://doi.org/10.1177/0261927X211044799

Valladolid, V. C. (2021). The role of coping strategies in the resilience and well-being of college students during the COVID-19 pandemic. Philippine Social Science Journal, 4(2), 30-42. https://doi.org/10.52006/main. v4i2.342

Yacat, J. A. (2017). Walang pakisama o walang kapwa-tao?: Isang sikolohikal na pagsusuri sa mga paglabag ng ibang tao at hindi ibang tao. Diwa E-Journal, 5, 1-22.

\section{Correspondence:}

PAUL DANIELLE P. LABOR*

pplabor@up.edu.ph https://orcid.org/0000-0001-9557-2869

MARIA CECILIA C. GASTARDO-CONACO

mcgastardoconaco@up.edu.ph

https://orcid.org/0000-0003-2064-2038

${ }^{*}$ Corresponding Author 